\title{
Comprehensive use of IT solutions to monitor the state of construction sites
}

\author{
Alexander Konikov* and Nikolay Garyaev \\ Moscow State University of Civil Engineering, Yaroslavskoe shosse, 26, Moscow, 129337, Russia
}

\begin{abstract}
The work examines new IT - solutions for monitoring the state of construction projects. Two areas are investigated: the first - monitoring of high-rise buildings and other large-scale objects, the second monitoring of construction sites located in remote, hard-to-reach areas. The possibilities of integrated use of the following information technologies are considered: Machine Vision, Wireless Sensor Network WSN, Edge Computing. Other new IT solutions are also in the field of view of the study: Cloud Computing, the Internet of Things, etc. To improve the quality of monitoring, it is proposed to use advances in other subject areas - in the field of unmanned aerial vehicles (drones) and in the space area (geostationary satellite communications). The idea of using drones and artificial earth satellites to monitor objects (particularly buildings) is not new in itself, but the use of these devices as part of the proposed set of IT technologies allows to bring solutions in the construction industry to a qualitatively higher level.
\end{abstract}

\section{Introduction}

Recently, there have been effective directions in IT technologies: Cloud Computing, Edge Computing, Wireless Sensor Network WSN, Internet of Things, Machine Vision, Deep Learning, Big Data, etc. It is interesting to explore the possibility of using these IT technologies in conjunction with advances in other areas of the economy - in the field of unmanned aerial vehicles (drones) and in the space field (geostationary satellite communications). Two specific areas in the construction industry have been proposed, in which the combination of these technologies allows to get good results. First, it is monitoring of high-rise buildings and other large-scale construction projects, and secondly monitoring of construction sites located in remote, not convenient for transport areas with poorly developed communications infrastructure. In both cases, it is possible to obtain innovative solutions and bring automated monitoring of buildings to a qualitatively higher level.

\footnotetext{
* Corresponding author: a.konikov@gmail.com
} 


\section{Materials and Methods}

Let's consider the problem of monitoring the state of high-rise and other large-scale construction projects. The proposed approach focuses on three modern IT technologies: Machine Vision, Wireless Sensor Networks WSN and Edge Computing. Let's first consider the first of them - Machine Vision.

In IT technologies there are two similar concepts: machine vision and computer vision $[1,2]$. However, there is a certain difference in the interpretation of these concepts. Machine Vision is the use of computer vision for production purposes. While Computer Vision is a common set of methods that allow computers to see. There are a number of applications in the construction industry where the use of machine vision seems very promising. These areas include: smart house, smart city, quality control of welds, use of unmanned aerial vehicles (drones) to control construction sites, etc. The paper examines these areas in the context of their use to monitor the condition of high-rise buildings and other large-scale construction projects.

Machine vision is a rather complex engineering solution that includes a number of components. First of all, the machine vision system should include one or more cameras. In the creation of machine vision systems are increasingly used so-called "smart cameras" that have in its composition an invader of frames, a specialized processor, etc. The complex "machine vision" includes software and hardware of various types. The equipment usually includes an ADC analog-digital converter that serves to digitize the image, a computer with quite powerful computational resources - is an obligatory component of the machine vision system. It is a specialized microprocessor that allows to speed up the performance of typical digital signal processing tasks: digital filtering, Fourier's transformation, signal search, etc. As far as software is concerned, specific applications are used to process images and detect properties in addition to conventional software. The complex "Machine Vision" often includes specialized light sources (LEDs, fluorescent and halogen lamps, etc.).

Machine vision can be successfully used in the construction industry. In the context of this study, first of all it should be noted directions: control of the quality of welded products and control of the cracks of the building. First, let's look at the first direction. The basis of many construction structures are welded structures: beams, farms, columns, masts, frames, reinforcement nets. The quality of welded structures is subject to strict requirements, regulated by the relevant regulations. Visual analysis of the welding joint allows to reveal welding cracks, and undercuts, pores, fistulas, burns etc. Most of these defects are unacceptable and need to be corrected. Visually-measuring non-destructive quality control of welds in construction structures has been carried out before, but the introduction of machine vision systems brings this work to a qualitatively higher level.

Now about the second direction - control the state of cracks of the building. Cracks in building structures can indicate a serious threat to the structure of the building and the safety of people. A variety of tools are used to control the cracks. For example, you can do this with special devices that act as sensors.

To monitor the quality and condition of welds, control the condition of cracks and other deviations from building codes, you can apply a machine vision system built on the basis of an unmanned aerial vehicle (drone). This direction is very promising in the construction and control of the state of large-scale construction sites: high-rise buildings, bridges, etc. For example, using machine vision mounted on a drone, you can explore areas of the building that are at high altitude hard-to-find parts of the bridge (Figure1). 

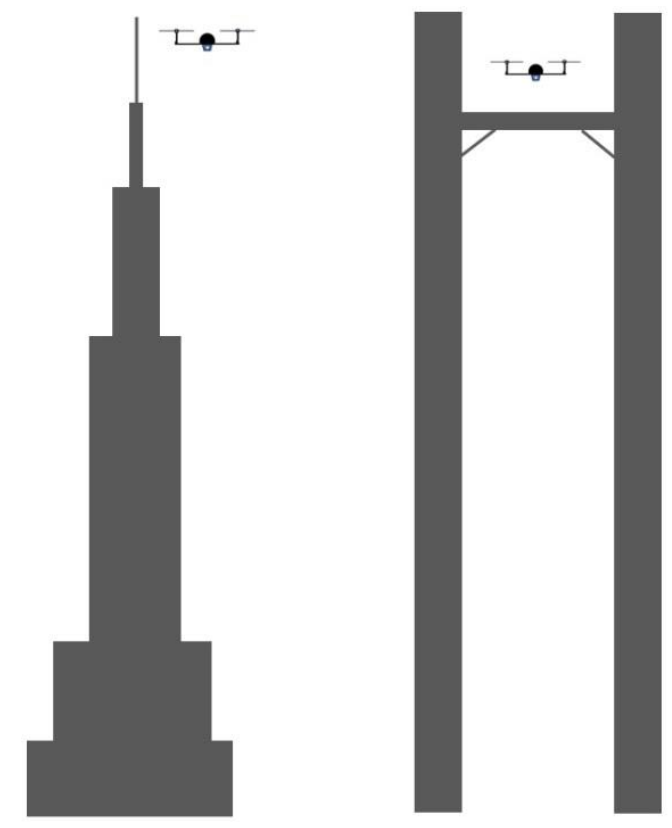

Fig. 1. Using machine vision installed on a drone, high-rise buildings are examined.

The idea of using drones for visual control of various objects is not new, such a solution is used in the construction industry. However, in known sources, the machine vision system is loosely tied to other modern IT systems, which severely limits its functionality.

In this work, the machine vision system is used in conjunction with other IT systems, primarily with a system of wireless sensors.

A few words about the sensors used to monitor the condition of a building. In the process of automated monitoring of the building, it is customary to use certain types of sensors. First of all, these are tensometric voltage sensors in the structural elements of the building. Tensometric motion sensors allow measuring the deformation at the point of installation to monitor surface cracks and joints in concrete structures (tunnels, piles, bridges, foundations, dams), as well as moving structural elements relative to each other. Tilt sensors (inclinometers) are used for long-term measurements of changes in the position of different structures, determining the size of bends and deformations of supports and beams. The list of sensors is continuously replenished: a separate set of sensors (humidity sensors, etc.) is used to conduct climate control in large buildings; another set of sensors are used in the Smart Home solution: water leak sensors, gas, penetration sensors, etc. The examination of the sensors can be continued, and it can be argued that a large number of sensors of different types located at different points in the building are required to monitor the condition of the building.

There is now a trend towards wireless methods of transmitting information from sensors. The benefits of wireless connectivity are well known - you don't need wires, you can move the sensor position mobility. The first advantage is particularly important for building monitoring, as many sensors must be located in places where wired communication is virtually impossible. At the same time there is a problem with energy consumption - we need low-cost sources of electricity, able without additional maintenance to maintain the health of the sensor for a long time. Hence the following conclusion: building monitoring requires wireless sensors with a small transmitter power, hence having a limited range. 
It should be clarified: under the sensor (Sensor Node) we will understand a set of devices, including, in addition to the sensor itself, ADC, digital computer (microcontroller), memory, power device and device providing wireless communication (receiver). Often, several sensors (temperature, humidity, etc.) are included in one sensor to expand functionality.

Pre-processing information from the sensor by a microcontroller allows to optimize the entire process of processing information. When designing automated monitoring devices, you should consistently take a system approach that takes into account the parameters of all the devices involved in the processing process, from Sensor Node to the data center. For example, if the ADC is incorrectly selected in a Sensory Node, it is difficult to compensate for subsequent processing.

To work effectively with wireless sensors, they are usually combined into the Wireless Sensor Network WSN [3-5]. The Sensor Nodes of one location area of WSN are connected to a Gateway that communicates with external devices and can pre-process information. The diagram of a typical WSN [3] is shown on Figure 2.

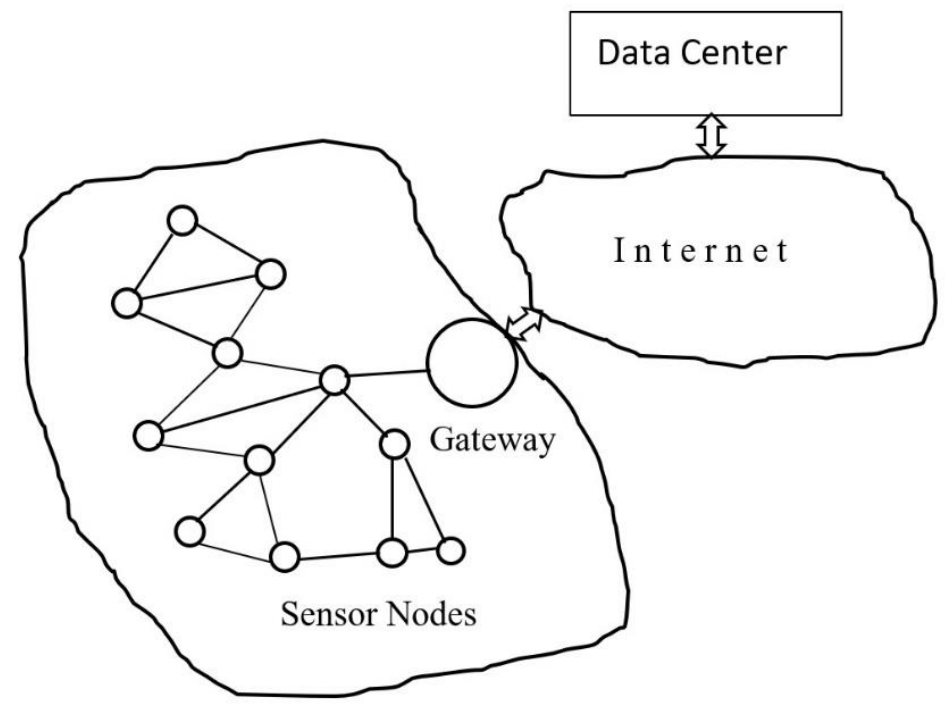

Fig. 2. The diagram of a typical Wireless Sensor Network

The issues of building a Wireless Sensor Network are well researched and addressed within the framework of the "WSN" concept - a distributed, self-organizing network of many sensors and executive devices combined by a radio channel. This technology is governed by standard 802.15.4 and is one of the most promising areas for the development of distributed surveillance and management systems for resources and processes. Therefore, we will not elaborate on the general issues concerning WSN, consider some of the features of use in the construction industry.

Sensors at the construction site are unevenly distributed: in certain areas of construction there are many of them, in others - not at all. It should be noted that the distances between localization zones in high-rise buildings and other large construction sites can be quite significant. These zones can be located in such a way that the most rational option for information sharing (from a technical and economic point of view) is the use of drones (Figure 3). 

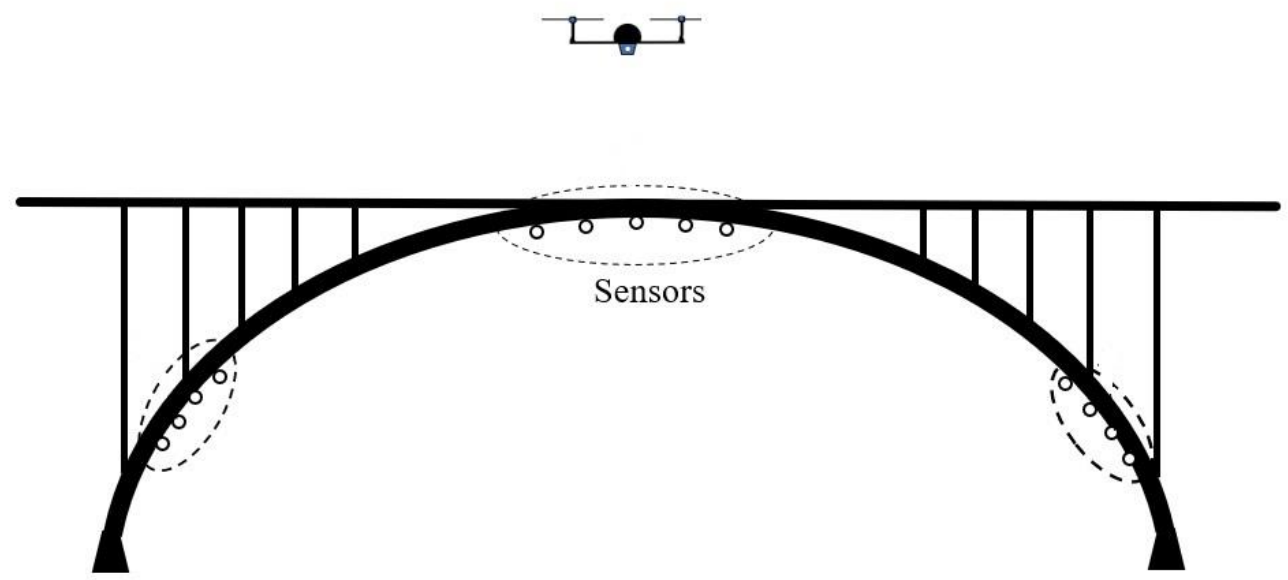

Fig. 3. The system that includes a drone and WSN

In economic calculation, it should be taken into account that drones are used not only to work with wireless sensors, but also include elements of the machine vision system, which visually analyzes the building.

Next, consider how to process information from the drone and other sources. The most common approach to processing is that the information collected is transferred to the Data Center, which is most often found "in the cloud" [6] (one of the variants of this approach is shown in Figure 2). However, this solution is not optimal, a more rational approach is to combine remote (cloud) computing and on-site processing. In our opinion, we should use a solution based on the "Edge Computing" concept [7,8]. According to this concept, a substantial portion of the information is processed "on the spot" while only the necessary information is provided to the data center of the entire system (which is most often in the "cloud").

There are prerequisites for this technique:

The automated monitoring system processes data from drones in conjunction with data obtained in other ways: sensors of different types, cameras, IoT devices, etc. Among the devices mentioned, IoT devices are particularly noteworthy, as due to the successes in the field of the Internet of Things $[9,10]$, there is a mass introduction of them. As a result, the amount of information generated is very large, and information can accumulate in real time. The flow of raw information is difficult to transmit to a remote center (even with a very broadband communication channel). Therefore, it is necessary to carry out pre-processing on the spot, which will significantly reduce the load on traffic to the external channel.

Another argument for using Edge calculations is that in some cases the time interval required to send data to a remote center, process data, and get results is unacceptably long. It requires the rapid processing and analysis of the data obtained "on-site" and the provision of results to employees working directly at the site.

Note that the use of Edge Computing in conjunction with Cloud Computing can be used very productively for intelligent information processing (Data Mining, Big Data, etc. [1117]).

Thus, a comprehensive method of automated monitoring of the state of high-rise and other large-scale construction projects has been proposed. The method is based on a number of new IT technologies, focusing on technology: Machine Vision, Wireless Sensor Networks and Edge Computing. The monitoring system includes an unmanned aerial 
vehicle (drone), which is especially important when monitoring highly located and hard-toreach areas of the building. The method allows to bring to a qualitatively different level monitoring of construction sites of this type.

Now let's consider another option of monitoring the construction site - when this facility is located in a remote, hard-to-reach area, with an underdeveloped communication infrastructure. In this case, you can use a set of IT solutions, including a geostationary satellite of the earth, to solve the issue[19]. The corresponding technical solution is shown on Figure 4.

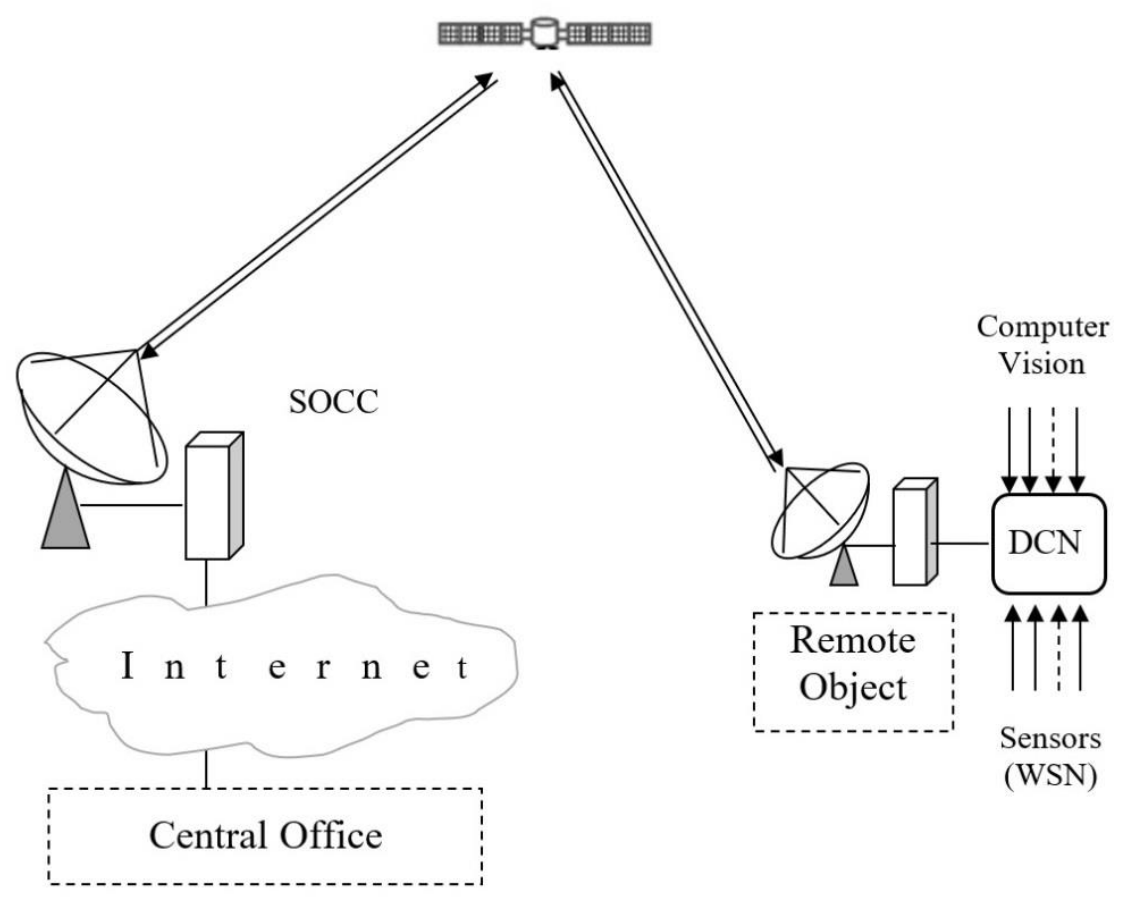

Fig. 4. Scheme of monitoring the state of construction remote object using Satellite technology

Signals about the state of a remote construction site are formed using the Data Collection System (DCN). The basic information comes from a Computer Vision system (in the simplest case it may consist of one or several video cameras) and sensors (may include a Network of Wireless Sensors). The signal from the satellite terminal located at the remote construction site is sent to a geostationary satellite, where it is processed and redirected to the satellite operator's control center Satellite Operator Control Centre SOCC, which is connected to global networks. Information from SOCC is sent via the Internet to the Central Office of the company that monitors the remote facility. This office can be located at a very long distance from both SOCC and a remote construction site.

Let us point out that one geostationary artificial satellite of the earth covers almost the entire territory of the Russian Federation. This territory includes areas where it is either impossible or economically impractical to communicate in another way. Therefore, in some cases there is simply no alternative to the use of satellite communications. There are a number of factors that can reduce the financial costs of such a system. One is that several remote construction sites can be connected to the satellite system, after which the corresponding unit costs for the facility are reduced. Another option is to temporarily monitor the facility. Satellite equipment installed at a remote site can be dismantled and 
transferred to another object. You can reduce costs by optimizing communication sessions with a remote object.

It is noted that there are a number of factors that can reduce the financial costs of the proposed system. One is that several remote construction sites can be connected to the satellite system, after which the corresponding unit costs for the facility are reduced. Another option is to temporarily monitor the facility. Satellite equipment installed at a remote site can be dismantled and transferred to another object. Costs can be reduced by optimizing remote-object communication sessions.

Note another point: the satellite channel can provide a duplex (two-track communication), so you can not only receive data from a remote construction site, but also transmit information to executive devices. This allows you to adjust the parameters that characterize the condition of the object (e.g. humidity or temperature inside the building) within the required boundaries.

Thus, this it-based complex provides remote control and maintenance of the construction site located in a remote area with poor communication infrastructure.

\section{Results}

The work offers a comprehensive solution to the problem of automated monitoring of highrise buildings, large bridges and other large-scale objects. Three modern IT technologies are adopted as the main ones: Machine Vision based on unmanned aerial vehicles (drone), Wireless Sensor Networks and Edge Computing. Other trends in IT technologies have been taken into account: Cloud Computing, the Internet of Things, Big Data technology.

The possibilities of "machine vision" technology have been investigated. It is noted that machine vision is a rather complex engineering solution, which includes a number of components. First of all, the machine vision system should include one or more cameras, other components in particular a specialized microprocessor, which allows to speed up the performance of typical tasks of digital signal processing.

There are areas in the construction industry where the machine vision system can be used. Two areas are highlighted: quality control of welding products and control of cracks of the building. Solving these problems is especially important for highly located areas of buildings, bridges, etc. because it is very difficult to get results in other ways.

It is noted that the systems of machine vision installed on the drone and used in construction are known. However, such systems are loosely tied to other modern IT systems, which severely limits functionality. It is proposed to use the machine vision system installed on the drone in conjunction with other IT systems, specifically - with wireless touch network Wireless Sensor Networks WSN.

A comprehensive solution based on the combination of the two systems has been proposed. To do this, the drone must be equipped with additional hardware and software. The solution has a number of important advantages, of which the main thing is that a comprehensive assessment of the state of the large-scale construction facility is carried out.

The question of how to process information received from a drone has been investigated. There are many options, including cloud-based processing. It is noted that the most rational way - the use of a solution based on the concept of "Edge Computing" is the most rational. The concept involves a significant portion of on-site processing, with only the necessary information provided to the entire processing center of the system (which is most often in the cloud). Arguments were presented in favor of such a decision.

The second direction is considered: monitoring of the construction site, when this object is located in a remote, hard-to-reach area, with an underdeveloped communication infrastructure. In this case, you can use a set of IT solutions, including a geostationary satellite of the earth, to solve the issue. 
Let us point out that one geostationary artificial satellite of the earth covers almost the entire territory of the Russian Federation. This territory includes areas where it is either impossible or economically impractical to communicate in another way. Therefore, in some cases there is simply no alternative to the use of satellite communications.

It is indicated that the satellite channel can provide duplex (two-way communication), so it is possible not only to receive data from a remote construction site, but also to transmit control information for executive devices. As a result, this IT-funded complex provides remote control and maintenance of the construction site located at a great distance from the company's main office in an area with poor communications infrastructure.

\section{References}

1. Computer vision: technologies, market, perspectives. TADVISER.

Government.Bisiness.IT. 2019. №6 - 26. https:// www.tadviser.com

2. Lukyanica A.A, A.G. Shishkin. A.G. Digital video processing. M.:, “AY-ES-ESPRESS". - 2009. 518 p.

3. Lihtcinder B.J., Kirychek R.V., Fedotov E.D. and oth. Wireless Sensor Networks. M.: "Hot Line-Telcom".2020.236p.

4. Tandel R.I. Learh Protocol in Wireless Server Network. A Survay // International Jurnal of Computer Science and Information Technologies. 2016.Vol. 7(4). P. 1894 1896

5. Powlak R.,Vojceichowski R.,Nicodem M/ New Simplified HEED Algorithm for Wireless Sensors Network // Computer networks, 17th conference, CN 2010, Ustron, Poland, June 15-19, 2010

6. Maximov K.V. The effectiveness of the use of cloud computing: methods and models of evaluation //Applied computer science, 2016. № 1(81), p.106-113.

7. Pedro Garcia Lopez, Alberto Montresor, Dick Epema, Anwitaman Datta, Teruo Higashino. Edgecentric Computing: Vision and Challenges // ACM SIGCOMM Computer Communication Review. 2015 .Vol. 45. iss. 5. pp. 37-42.

8. Peripheral calculations (Edge computing). TADVISER. Government.Bisiness.IT. 2019. №11 - 7. https:// www.tadviser.com

9. Olivier Hersent, David Boswarthick, Omar Elloumi. The Internet of Things: Key Applications and Protocols. - Willey, 2012. - $370 \mathrm{p}$

10. L.Chernyak. IoT platform. Open systems. DBMS, 2012. № 7.

11. Alexandr Konikov, Ekaterina Kulikova and Olga Stifeeva. Research of the possibilities of application of the Data Warehouse in the construction area. MATEC Web of Conferences 251, 03062 (2018)

12. Konikov A., Konikov G. Big Data is a powerful tool for improving the environment in the construction business. IOP Conference Series: Earth and Environmental Science, 2017, vol. 90, p. 012184.

13. Konikov A.I. Promising areas in the field of information systems for construction management // Industrial and Civil Engineering, 2019, №6, p. 64-69

14. A.I. Konikov. Study of a number of aspects of using Big Data technology in constructione, BST Journal, 2019, №2, p. 28-29.

15. Nikolay Ivanov and Maxim Gnevanov. Big data: perspectives of using in urban planning and management. MAT EC Web of Conferences 170, 01107 (2018)

16. Kurt Stockinger, Nils Bundi, Jonas Heitz and Wolfgang Breymann. Scalable architecture for Big Data financial analytics: user-defined functions vs. SQL. Journal of Big Data. March 2019.DOI10.1186/s40537-019-0209-0 
17. Gnevanov M. V., Ivanov N. A. Big Data technology - using in urban planning // Industrial and Civil Engineering, 2018. № 4. p. 83-87.

18. Valpeters M., Kireev I., Ivanov N., 2018. Application of machine learning methods in big data analytics at management of contracts in the construction industry. MATEC Web of Conferences, 170, 01106

19. Konikov A. Promising wireless applications in the construction industry. E3S Web of Conferences 164, 10043 (2020) TPACEE-2019

20. Evstratov V. Some aspects of intelligent decision support systems in construction // IOP Conference Series: Materials Science and Engineering Volume 1030, Issue 1, 14 January 2021, 012066 